\title{
A Centralized Shifted Voltage Control Method for Accurate Power Sharing in DC Islanded Microgrids
}

\author{
Minh-Duc Pham and Hong-Hee Lee \\ Department of Electrical Engineering \\ University of Ulsan, Korea \\ Phone/Fax number:+82-52-259-2187, e-mail: minhducpham2009@gmail.com, hhlee@mail.ulsan.ac.kr
}

\begin{abstract}
Due to line impedance mismatch among renewable energy sources (RESs), it is hard to realize accurate power sharing in the DC microgrid system. To solve this issue, a distributed power sharing strategy for adjusting the RES output voltage is developed by adding shifted output voltage into each local controller. Thanks to the shifted voltage, the influence of voltage drop caused by the droop controller is effectively mitigated, so that the DC bus voltage is constantly balanced regardless of the load changes. The proposed method is realized with a centralized approach, and all the required control variable to determine the reference voltage is transmitted through low-bandwidth communication. The controller design and system stability are analyzed in detail with a simplified microgrid model. Small-scale DC microgrid is simulated to verify the effectiveness of the centralized shifted voltage control method.
\end{abstract}

Keywords. DC microgrid, Centralized control approach, Proportional power sharing.

\section{Introduction}

DC microgrid is well known as a reliable solution to integrate renewable energy sources and power generators into rural areas [1]. A microgrid is a small-scale system that integrates RES units such as photovoltaic, wind turbine, and energy storage system. Microgrids have become a widely accepted concept for the superior connection of RESs in power networks. Compared to the traditional power systems, DC microgrid has better efficiency in transmission and distribution because the reactive power is neglected [2]. Furthermore, the harmonic issue and the voltage quality can be easily improved by controlling the DC microgrid. With these advantages, the DC microgrid is concerned these days [3], [4].

Fig. 1 shows a typical DC microgrid configuration, where all RESs and loads are connected to DC bus. The microgrid central controller (MGCC) monitors DC bus voltage and all RESs' condition for protection and power demand assignment. Since all the RESs are decentralized in the microgrid, the interfacing DC-DC converters are connected in parallel, as shown in Fig. 1. The controller of power converter is a key issue for effective load power sharing among different RESs. Many control methods have been proposed to achieve proper power sharing in a parallel

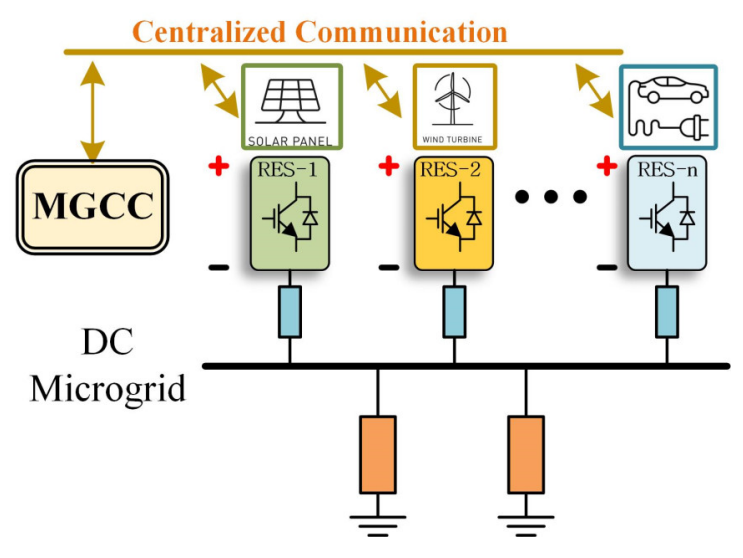

Fig. 1 DC microgrid configuration.

DC-DC converter system, such as master-slave control [5], circular current control [6], and droop control method [7], [8]. Among these methods, the droop control algorithm is commonly adopted as an efficient power sharing method due to its simplicity, low cost, and autonomous characteristics [9]. The droop controller needs only local measurement to adjust the output voltage of the RES converter according to its output current [10]. Although the droop control method can extend to a large number of RES with low computation, the influence of the line impedance on the output voltage drops still has not been considered sufficiently [11]. Hence, it is impossible to share the load power proportionally among the RESs since the line impedance value is not the same in practical DC microgrid.

To address the current sharing inaccuracy in DC microgrids, a control scheme based on the average DC output current in each of the converters was proposed [12]. Although the DC bus voltage quality is improved, the enhancement of power sharing was not obvious because only average value is considered. To address this problem, a circulating current control method is proposed in [13], which is based on no-load circulating current values. On the other hand, the proposed adaptive droop resistance is proposed in [14], which varies the droop resistance to track the load current variation. Nevertheless, the output voltage 
quality is reduced significantly because of the noticeable voltage drop due to the high value of droop resistance.

In the improved droop control method discussed in [15], conventional droop control is used to achieve load current sharing with the aid of low bandwidth communication. But, the system response is slow because of the delay among converters. A cooperative algorithm with a voltage regulator and the current regulator is presented with enhanced power sharing response [16]. In this algorithm, the current regulator compares local per-unit power with the neighbors' per-unit, and accordingly adjusts the droop virtual impedance to balance the power sharing among RESs. However, the power sharing algorithm still has a poor voltage regulation.

To overcome the conventional control drawbacks, a centralized shifted voltage control method is presented for RESs in the DC microgrid. In the proposed controller, a controllable shifted voltage is added to each local controller for RES output voltage regulation. By adaptively adjusting the shifted voltage, the voltage drop caused by the droop controller is mitigated, and the power sharing is accurately achieved. Also, the power sharing performance is maintained under load variation. The detailed analysis and design procedure are clearly described for two DC-DC buck converters connected in parallel.

\section{Power Sharing in DC Microgrid}

To simplify the DC microgrid system, a single line circuit of two parallel DC-DC converters are considered in Fig. 2, where $V_{D C 1}, V_{D C 2}, I_{O 1}, I_{O 2}, R_{\text {line } 1}, R_{\text {line } 2}, r_{d 1}$, and $r_{d 2}$ represent the converter voltages, output currents, line impedance, and droop impedance of converter-1 (RES1) and converter-2 (RES2), respectively. By assuming that the power rating and converter parameters of RES1 and RES2 are identical, the ratio of the output power per unit of two RESs $\left(p_{p u 1} / p_{p u 2}\right)$ is derived from microgrid circuit in Fig. 2:

$$
\begin{aligned}
\frac{p_{p u 1}}{p_{p u 2}} & =\frac{P_{1}}{P_{2}}=\frac{\left(R_{\text {line } 1}+R_{\text {load }}\right) I_{O 1}{ }^{2}}{\left(R_{\text {line } 2}+R_{\text {load }}\right) I_{O 2}{ }^{2}}, \\
& =\frac{-r_{d 1} I_{O 1}{ }^{2}+V_{D C 1} I_{O 1}}{-r_{d 2} I_{O 2}{ }^{2}+V_{D C 2} I_{O 2}}
\end{aligned},
$$

where $R_{\text {load }}$ is the load resistance. From (1), when the output current is changed if the load is varied, the power sharing between RES1 and RES2 becomes disproportional. In order to address this issue, a proper compensation of line impedance or converter voltage regulation is required to achieve the proportional power sharing in (1). As we know, it is generally hard to detect the line resistance in practical applications. In stead of the line resistance, if $V_{D C 1}$ and $V_{D C 2}$ in (1) are chosen correctly, the accurate power sharing can be ensured despite the load condition changes suddenly.

\section{Proposed Control Method}

\section{A. Primary Voltage Control}

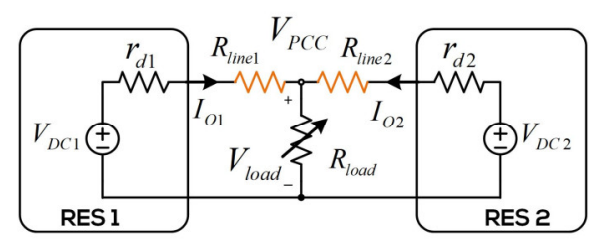

Fig. 2 Parallel DC-DC converters with different output voltages.

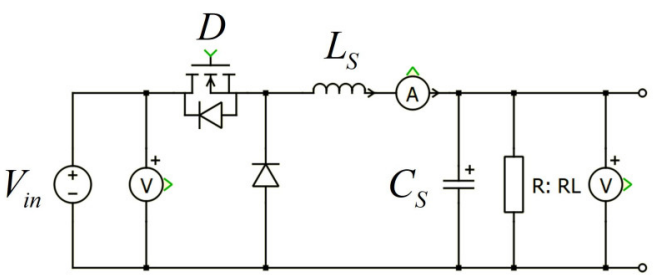

Fig. 3 Simplified circuit of Buck converter.

To ensure a voltage regulation for all RES units, the droop control algorithm is applied for the primary control loop. The droop controller determines the virtual voltage drop across the virtual impedance for each DG. The droop impedance is emulated by taking the output current feedback from the $\mathrm{i}^{\text {th }}$ converter and multiplied by the droop impedance $r_{d i}$. The voltage drop on the virtual impedance is then subtracted from the nominal voltage of each converter, and the converter reference signal is generated as

$$
V_{D C d r i}=V_{n o m}-I_{O i} r_{d i},
$$

where $V_{\text {nom }}$ is the converter nominal voltage, which is equal to that of the DC bus nominal voltage. By using (2), the load power is autonomously shared among RESs.

\section{B. Shifted Voltage Control Method}

In order to improve the power sharing performance, the voltage difference among converters has to be suppressed. To satisfy this control objective, an additional shifted voltage is added into the reference in (2). Then, the new voltage reference is obtained as following:

$$
V_{D C r e f}^{*}=V_{D C d r i}+V_{\text {Shift }}=V_{\text {nom }}-I_{O i} r_{d i}+V_{\text {Shift }}
$$

where $V_{D C \text { ref }}^{*}$ is the converter voltage reference, and $V_{\text {Shift }}$ is the shifted voltage.

In order to determine the shifted voltage, the output power per unit of each DG is identified through low-bandwidth communication. Then, the average power supply of all DGs is obtained:

$$
\begin{gathered}
P_{p u i}=P_{i} / P_{\text {ratei }}, \\
\overline{P_{p u i}}=\frac{1}{n} \sum_{i=1}^{n} P_{p u i},
\end{gathered}
$$

where $\overline{P_{p u i}}$ is the average power demand of all RESs, which is calculated by the MGCC. Then, the value of $\overline{P_{p u i}}$ is sent to all RESs in the microgrid through the lowbandwidth communication. 


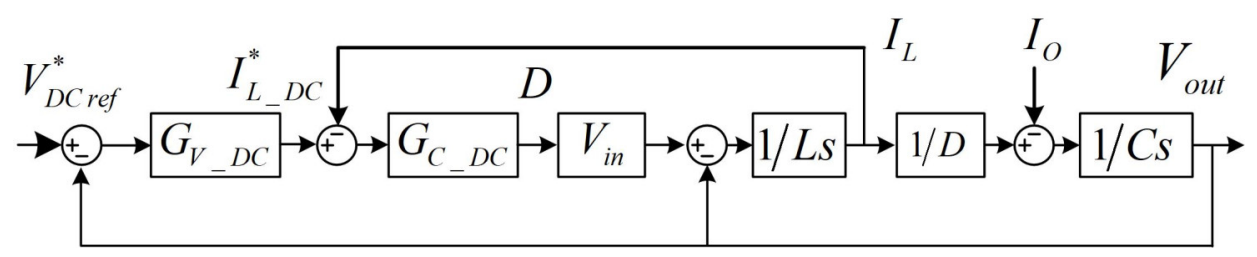

Fig. 4 Control diagram of a single RES converter.

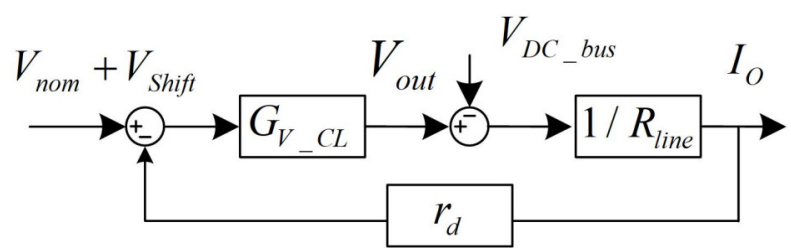

Fig. 5 Droop control diagram with the shifted voltage part.

The local controller regulates the shifted voltage using the following proportional integral controller:

$$
V_{\text {Shift }}=K_{P_{-} p u}\left(\overline{P_{p u i}}-P_{p u i}\right)+K_{I_{-} p u} / s\left(\overline{P_{p u i}}-P_{p u i}\right),
$$

where $K_{P_{-} p u}$ and $K_{I_{-} p u}$ are the controller gains. In (6), the shifted voltage is obtained by means of the only simple proportional integral controller, and the calculation burden of the distributed controller is much reduced.

\section{Controller Design and Stability Analysis}

\section{A. Voltage Control Loop}

A RES converter in the DC microgrid is implemented based on Buck converter in Fig. 3. A double loop controller is employed for each RES converter with the voltage reference calculated from (3) for good tracking performance. The outer voltage loop generates the inductor current reference, and the inner current loop regulates the output PWM duty to track its current reference. The control diagram of the single RES converter with a double loop controller is shown in Fig. 4.

The inner current control loop is derived from Fig. 4:

$$
\left[G_{C_{-} D C}\left(I_{L}-I_{L_{-} D C}^{*}\right) V_{\text {in }}-V_{\text {out }}\right] /(L s)=I_{L},
$$

To design the controller gain, the open-loop and closedloop transfer functions of the current controller are obtained as following:

$$
\begin{gathered}
G_{I_{-} O L}=\left(V_{\text {in }} G_{C_{-} D C}\right) /(L s), \\
G_{I_{-} C L}=\left(V_{\text {in }} G_{C_{-} D C}\right) /\left(V_{\text {in }} G_{C_{-} D C}+L s\right),
\end{gathered}
$$

where $G_{C D C}$ is the current controller, which is expressed as

$$
G_{C_{-} D C}=K_{P C}+K_{I C} / s,
$$

where $K_{P C}$ and $K_{I C}$ are the gains of $G_{C_{-} D C}$. The inner current control loop bandwidth of $G_{C_{-} D C}$ is set as $1 / 20$ of the switching frequency $\left(f_{s w}\right)$. To ensure the system stability, the phase margin has to be higher than $50^{\circ}$, and the gains $K_{P C}$ and $K_{I C}$ of $G_{C_{-} D C}$ are designed to satisfy the following condition:

$$
\begin{aligned}
& G_{I_{-} O L}\left(j \omega_{\text {cut-off }}\right)=\left|V_{\text {in }}\left[K_{P C}+K_{I C} /\left(j \omega_{\text {cut }- \text { off }}\right)\right] /\left(L j \omega_{\text {cut }- \text { off }}\right)\right|=1 \\
& \angle G_{I_{-} O L}\left(j \omega_{\text {cut }- \text { off }}\right) \geq 50^{\circ}
\end{aligned}
$$

where $\omega_{\text {cut-off }}=2 \pi f_{\text {cut-off }}$, and $f_{\text {cut-off }}=(1 / 20) f_{\text {sw }}$. The gains $K_{P C}$ and $K_{I C}$ from (11) and (12) are simplified as

$$
\begin{aligned}
K_{P C} & =\omega_{\text {cut }-o f f} L / V_{\text {in }}, \\
\frac{K_{I C}}{K_{P C}} & =G_{\text {damp_C }} \omega_{\text {cut-off }},
\end{aligned}
$$

where $G_{\text {damp_C }}$ is the damping gain which is selected to ensure that the phase margin of $G_{C_{-} D C}$ complies with the condition in equation (14). On the other hand, the outer voltage control loop is derived as

$$
\left\{\left(V_{\text {in }}-V_{\text {Out }}\right) G_{V_{-} D C} G_{I_{-} C L} / D-I_{O}\right\} /(C s)=V_{\text {out }},
$$

where $G_{V D C}$ is the voltage controller, which is expressed as

$$
G_{V_{-} D C}=K_{P V}+K_{I V} / s,
$$

where $K_{P V}$ and $K_{I V}$ are the gains of $G_{V_{-} D C}$. The outer voltage control loop bandwidth is slower than the current control loop to guarantee the controller stability. Similarly, we can calculate the gains $K_{P V}$ and $K_{I V}$ of $G_{V_{-} D C}$ as follows:

$$
\begin{gathered}
K_{P V}=D C \omega_{V C}, \\
\frac{K_{I V}}{K_{P V}}=G_{d a m p_{-} V} \omega_{V C},
\end{gathered}
$$

where $\omega_{V C}=2 \pi f_{V C}, G_{d a m p_{-} V}$ is the damping gain for good damping ratio. The designed parameters of the RES converter controller are listed in Table I.

\section{B. Centralized Control Loop}

Fig. 5 shows the droop control diagram with the shifted voltage. The output voltage of the $i^{\text {th }}$ RES is expressed as

$$
\begin{aligned}
V_{\text {out }} & =\left[V_{\text {nom }}+V_{\text {Shift }}-I_{O} r_{d}\right] G_{V_{-} C L} \\
& =V_{\text {nom }} G_{V_{-} C L}+V_{\text {Shift }} G_{V_{-} C L}-I_{O} r_{d} G_{V_{-} C L}
\end{aligned},
$$


Table I. DC Microgrid Parameters

\begin{tabular}{cc}
\hline \hline Parameters & Value \\
\hline Bus Voltage $\left(V_{\text {nom }}\right)$ & $24 \mathrm{~V}$ \\
Rated Power $\left(P_{\text {rated } 1-2}\right)$ & $1000 \mathrm{~W}$ \\
Droop coefficient $\left(r_{d 1,2}\right)$ & $0.2 \Omega$ \\
Line impedances $1 \& 2$ & $0.1 \Omega$, \\
$\left(R_{\text {line } 1,2}\right)$ & $0.2 \Omega$ \\
Load $1 \& 2\left(R_{\text {load } 1,2}\right)$ & $2 \Omega$ \\
Switching frequency $\left(f_{\text {sw }}\right)$ & $20 \mathrm{KHz}$ \\
Output capacitor $(C)$ & $680 \mu F$ \\
Inductor $(L)$ & $340 \mu \mathrm{H}$ \\
Input Voltage $\left(V_{\text {in }}\right)$ & $35 \mathrm{~V}$ \\
\hline \hline
\end{tabular}

where

$$
I_{O}=\left(V_{\text {out }}-V_{D C_{-} \text {bus }}\right) / R_{\text {line }} .
$$

The second term $\left(V_{\text {Shift }} G_{V_{-} C L}\right)$ in (19) shows that the proposed shifted voltage can adjust the output voltage of the RES unit. On the other hand, the third term ( $\left.-I_{O} r_{d} G_{V_{-} C L}\right)$ has a negative sign which represents the unavoidable voltage drop caused by the droop control algorithm. Thanks to the proposed shifted voltage, the negative influence of the droop control is compensated so that the output voltage among RESs becomes balanced.

By assuming that the double loop voltage control is well designed, the voltage tracking loop transfer function $\left(G_{V_{-} C L}\right)$ can be regarded as a unity in case of designing the centralized control loop. Thus, the open-loop of the centralized controller is simplified as follows:

$$
G_{O_{-} \text {Central }}=\left(K_{P_{-} \text {Central }}+K_{I_{-} \text {Central }} / s\right)\left(\overline{P_{p u i}}-P_{p u i}\right) G_{V_{-} C L} \text {, }
$$

The bandwidth of $G_{O L_{-} \text {Central }}$ is equal to the minimum communication bandwidth between a centralized controller and RES for ensuring stability. Then, $K_{P_{-} \text {Central }}$ and $K_{I_{-} \text {Central }}$ are selected as 0.1 and 20, respectively, to provide $10 \mathrm{~Hz}$ open-loop cut off frequency.

\section{Simulation Results}

To evaluate the performance of the proposed centralized control method, a DC microgrid system with two RESs was simulated by means of PLECS 4.1 simulation software. The microgrid configuration used in the simulation is shown in Fig.6 with parameters listed in Table I. Each RES in DC microgrid composes of DC-DC buck converter with a double loop voltage controller as shown in Fig. 6.

Fig. 7 shows the performance of the DC microgrid with and without the proposed shifted voltage when only load 1 is connected. Before $t=t_{1}$, the output powers of RES1 and RES2 are highly different because the voltage drop does not match even though their power ratings are the same. When the proposed scheme starts to operate at $t=t_{1}$, the output power of each RES becomes the same value with $P_{1}=P_{2}$. The accurate power sharing is achieved

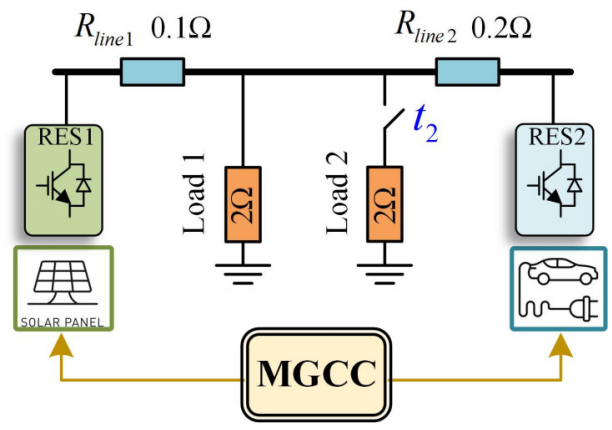

Fig. 6 DC microgrid used in simulation.

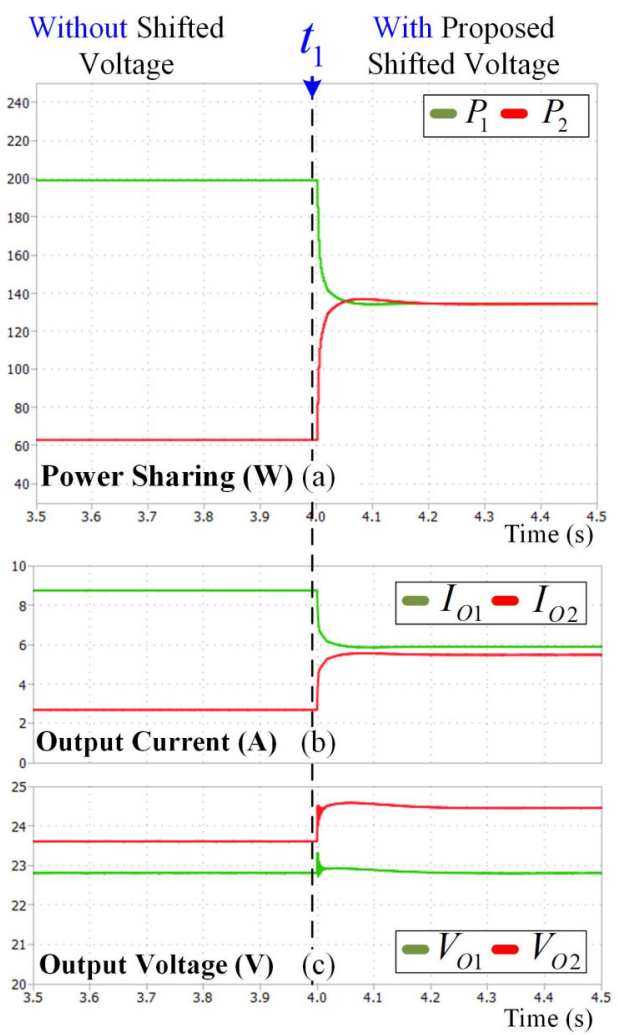

Fig. 7 Performance of the DC microgrid with and without the proposed shifted voltage. (a) power sharing (b) RES output current (c) output voltage.

thanks to the proper shifted voltage adjustment. During the proposed voltage adjusting, the circulating current is reduced significantly as shown in Fig. 7 (b). In addition, the output voltage magnitude of two RESs is not decreased, so that the DC bus voltage quality is guaranteed.

Fig. 8 shows the dynamic response for the load changes when load 2 is connected at $t=t_{2}$. The proposed control method keeps accurate power sharing despite the load is doubled. The output powers of two RESs reach their steady-state values without any fluctuation. Furthermore, the drop voltage caused by the load step change is recovered within a short transient time of $0.01 \mathrm{~s}$. From the simulation results in Figs. 7 and 8, the proposed 
control method verifies that the proportional load power sharing is well achieved regardless of the load variation.

\section{Conclusion}

In this study, a centralized control method was developed by inserting the shifted voltage into the local voltage control loop. The shifted voltage is adaptively adjusted by using the feedback power sharing error for output voltage regulation. By using the proposed shifted voltage technique, the voltage drop caused by the droop controller was effectively compensated to obtain proportional and accurate load power sharing. Despite doubling the load power, the proposed control method retains the correct power sharing without output voltage degradation.

\section{Acknowledgment}

This research was supported by Korea Electric Power Corporation. (Grant number: R20XO02-33)

This work was supported by the KETEP and the MOTIE under Grant 20194030202310.

\section{References}

[1] L. Che and M. Shahidehpour, "DC microgrids: Economic operation and enhancement of resilience by hierarchical control," IEEE Trans. Smart Grid, 2014.

[2] V. Nasirian, S. Moayedi, A. Davoudi, and F. L. Lewis, "Distributed cooperative control of dc microgrids," IEEE Trans. Power Electron., 2015.

[3] F. Nejabatkhah and Y. W. Li, "Overview of Power Management Strategies of Hybrid AC/DC Microgrid," IEEE Transactions on Power Electronics. 2015.

[4] Dong, I. Cvetkovic, D. Boroyevich, W. Zhang, R. Wang, and P. Mattavelli, "Grid-interface bidirectional converter for residential DC distribution systems - Part one: Highdensity two-stage topology," IEEE Trans. Power Electron., 2013.

[5] J. Rajagopalan, K. Xing, Y. Guo, F. C. Lee, and B. Manners, "Modeling and dynamic analysis of paralleled $\mathrm{dc} / \mathrm{dc}$ converters with master-slave current sharing control," 1996.

[6] Wu Tsai-Fu and Y. K. Chen, "3C strategy for inverters in parallel operation achieving an equal current distribution," IEEE Trans. Ind. Electron., 2000.

[7] S. K. Sahoo, A. K. Sinha, and N. K. Kishore, "Control Techniques in AC, DC, and Hybrid AC-DC Microgrid: A Review," IEEE Journal of Emerging and Selected Topics in Power Electronics. 2018.

[8] X. Lu, J. M. Guerrero, K. Sun, J. C. Vasquez, R. Teodorescu, and L. Huang, "Hierarchical control of parallel AC-DC converter interfaces for hybrid microgrids," IEEE Trans. Smart Grid, 2014.

[9] M. D. Pham and H. H. Lee, "Fuzzy PID controller for PCC voltage harmonic compensation in islanded microgrid," in Lecture Notes in Computer Science (including subseries Lecture Notes in Artificial Intelligence and Lecture Notes in Bioinformatics), vol. 10954 LNCS, Springer, Cham, 2018, pp. 231-242.

[10] M.-D. Pham and H.-H. Lee, "Improved Reactive Power Sharing and Harmonic Voltage Compensation in Islanded Microgrids Using Resistive-Capacitive Virtual Impedance," J. Power Electron., vol. 19, no. 6, pp. 1575 $1581,2019$.

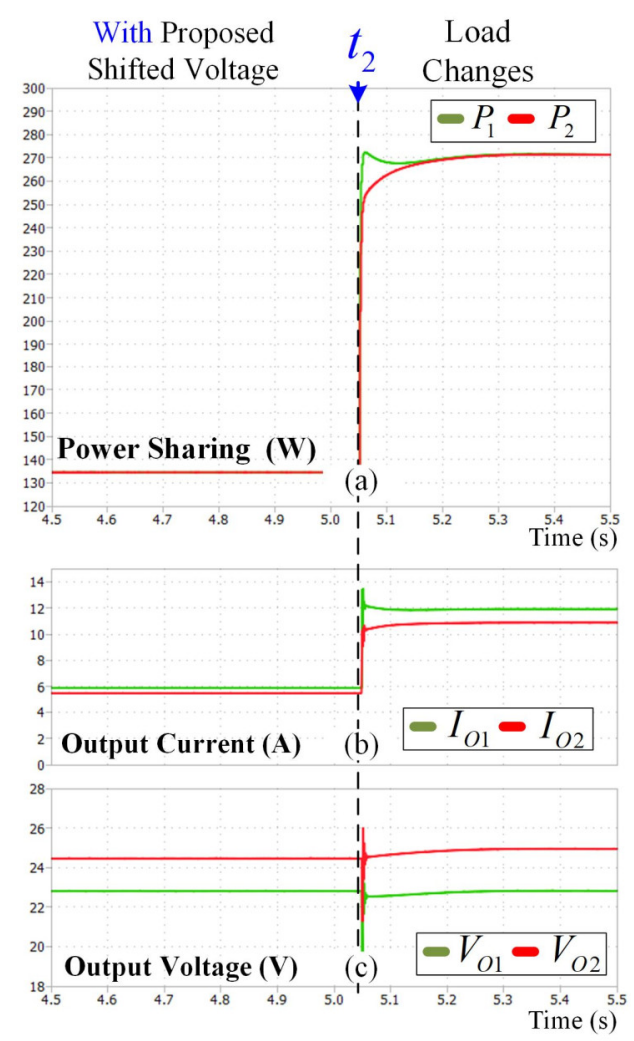

Fig. 8 Performance of the microgrid with the proposed shifted voltage during load changes. (a) power sharing (b) RES output current (c) output voltage.

[11] A. Khorsandi, M. Ashourloo, H. Mokhtari, and R. Iravani, "Automatic droop control for a low voltage DC microgrid," IET Gener. Transm. Distrib., 2016.

[12] S. Anand, B. G. Fernandes, and J. M. Guerrero, "Distributed control to ensure proportional load sharing and improve voltage regulation in low-voltage DC microgrids," IEEE Trans. Power Electron., 2013.

[13] S. Anand and B. G. Fernandes, "Modified droop controller for paralleling of dc-dc converters in standalone dc system," IET Power Electron., 2012.

[14] S. Augustine, M. K. Mishra, and N. Lakshminarasamma, "Adaptive droop control strategy for load sharing and circulating current minimization in low-voltage standalone DC microgrid," IEEE Trans. Sustain. Energy, 2015.

[15] X. Lu, J. M. Guerrero, K. Sun, and J. C. Vasquez, "An improved droop control method for de microgrids based on low bandwidth communication with dc bus voltage restoration and enhanced current sharing accuracy," IEEE Trans. Power Electron., 2014.

[16] W. W. A. G. Silva, T. R. Oliveira, and P. F. DonosoGarcia, "An Improved Voltage-Shifting Strategy to Attain Concomitant Accurate Power Sharing and Voltage Restoration in Droop-Controlled DC Microgrids," IEEE Trans. Power Electron., 2021. 